\title{
Journinal of Digital Imiaging
}

\section{Dual-isotope Acquisition for CT-SPECT Registration of Infection Studies}

\author{
Lisa Tang, ${ }^{1}$ Ghassan Hamarneh, ${ }^{1}$ and Anna Celler ${ }^{2}$
}

The registration of $\mathrm{CT}$ and NM images can enhance patient diagnosis since it allows for the fusion of anatomical and functional information as well as attenuation correction of NM images. However, irrespective of the methods used, registration accuracy depends heavily on the characteristics of the input images and the degree of similarity between them. This poses a challenge for registering CT and NM images as they may have very different characteristics. To address the particular problem of CT and In-111 SPECT registration, we propose to perform a dual-isotope study which involves an additional injection of Tc-99m MDP to generate two inherently registered images: In-111 SPECT and Tc-99m SPECT. As skeletal structures are visible in both CT and TC-99m SPECT, performing registration of these images may be much more effective. The very same spatial transformation derived can be immediately applied to complete the registration of CT and the corresponding In-111 SPECT. Accordingly, we hypothesize that the registration of CT and Tc-99m SPECT can be more accurately performed than the registration of CT and In-111 SPECT and seek to compare the accuracies between the aforementioned registrations. In this paper, we have collected three clinical datasets, with the ground-truth transformations known, and tested the proposed approach by using a mutual information-based algorithm to solve for the rigid/non-rigid misalignments introduced to them. Based on the results of our experiments, we conclude that registration using Tc-99m SPECT can achieve $100 \%$ success rate, and is thus much more superior to the registration using In-111 SPECT, which at best, achieves only $38 \%$ success rate. Clearly, the introduction of a dual-isotope acquisition can substantially improve the registration of SPECT and $\mathrm{CT}$ images.

KEY WORDS: 3D imaging (imaging, three-dimensional), image fusion, image processing, image registration, computed tomography, nuclear medicine, single photon emission computed tomography (SPECT), simulation, graphical user interface (GUI)

\section{INTRODUCTION}

S ingle photo emission computed tomography $N$ (SPECT) is a widely available clinical diagnostic technique used in the assessment of cardiac, renal, pulmonary, and other organ functions as well as the diagnosis of many diseases. Recently, SPECT has also been gaining importance in oncological diagnosis ${ }^{1,2}$ and radiotherapy planning $^{3}$. SPECT functional images, however, often lack anatomical information, which is necessary to localize disease sites $^{4}$. Further, SPECT suffers from poor resolution ${ }^{5}$ due to the effect of collimator blurring. In addition, their diagnostic and quantitative accuracies are affected by photon attenuation and scatter in the body ${ }^{6,7}$. Without proper attenuation and scatter correction, tumors located at different depths will show different radiotracer uptakes, leading to significant errors in diagnosis, internal dosimetry calculations, tumor staging, radiotherapy planning, and treatment evaluation.

Accurate registration and fusion of SPECT with computed tomography (CT) images will likely help to resolve these issues. As CT images contain detailed anatomical information, SPECT-CT fu-

${ }^{1}$ From the Medical Image Analysis Lab, Simon Fraser University, Burnaby, BC, Canada V5A 1 S6.

${ }^{2}$ From the Medical Imaging Research Group, University of British Columbia, Vancouver, BC, Canada V5Z 1L8.

Correspondence to: Lisa Tang, Medical Image Analysis Lab, Simon Fraser University, Burnaby, BC, Canada V5A 1S6; tel: +1-778-7825509; e-mail: lisat@sfu.ca

Copyright (C) 2009 by Society for Imaging Informatics in Medicine

Online publication 10 January 2009

doi: 10.1007/s10278-008-9171-7 
sion can provide localization information useful for the interpretation of SPECT data. Moreover, because CT measures tissue density distribution, the registered CT images can be converted into attenuation maps and be used for attenuation and scatter correction of the SPECT data ${ }^{5,6}$. Lastly, by incorporating information from the registered CT image into the SPECT reconstruction procedure, we may also improve the spatial resolution of $\mathrm{SPECT}^{8,9}$.

Numerous software-based methods for multimodal image registration have been proposed ${ }^{7,10,11}$; these are usually classified as image-, feature-, or surface-based approaches. Regardless of the methods employed, however, automatic techniques are only robust when both images contain identifiable and corresponding features ${ }^{12,13}$. Unfortunately, this condition is not always fulfilled. For example, when registering CT with SPECT, the functional information in SPECT often does not correspond to the anatomical information in CT. This situation is often seen in diagnostic oncology studies where tracer uptake is, by design, high at tumor sites but low or very low in other body regions (e.g., ${ }^{4}$ ).

The same situation may be observed when we perform registration of $\mathrm{CT}$ and SPECT images involving tumor and skeletal infections. To remedy this situation, we have proposed to replace the standard single-isotope SPECT study with a dualisotope acquisition ${ }^{14,15}$. However, as we could only test our method using data that consisted of independently acquired SPECT images, rather than dual-isotope studies, it is uncertain exactly how much value the additional tracer and its corresponding generated SPECT images bring to accurate registration. It is thus part of our goal to realize the proposed idea with existing clinical studies. In particular, we apply our method to diagnostic studies concerning skeletal infections. In these studies, In-111 is used to capture regions of possible infections or inflammations. With dualisotope imaging, Tc-99m will also be administered. As these two tracers emit photons at different energies, two separate but inherently registered images can be reconstructed from the same acquisition, generating an infection SPECT image and a bone SPECT image. As Tc-99m SPECT usually shows much more tracer uptake in the bone marrow than In-111 SPECT does, more visible and identifiable bone structures can be seen. Since these structures are also present and can be easily identified in the corresponding CT image, Tc-99m SPECT will contain more information that is similar and correlated to the information in CT than In-111 SPECT does. Consequently, we hypothesize that the registration of Tc-99m and CT images will be more accurate and robust than the registration of In-111 SPECT and CT. Example slices of these images in one of the collected chest studies are shown in Figure 1. There, we can see that In-111 SPECT (Fig. 1a) has fewer image features that are correlated to the skeletal structures captured in the CT image than Tc-99m SPECT (Fig. 1b) does. Accordingly, the objective of this work is to test the hypothesis that the use of In-111 SPECT from a dual-isotope acquisition increases accuracy of the registration of $\mathrm{CT}$ and infection SPECT by comparing registrations of three scenarios, each containing CT and either one of: (1) Tc-99m SPECT, (2) In-111 SPECT, or (3) a fusion of the two SPECT images. Through analysis of the results, we could then quantify the significance and amount of improvement, if any, dual-isotope acquisitions can bring to registration, and by which we justify its use.

The outline of the rest of the paper is as follows. We first describe the details of the data involved in this work in "Materials" section. We then formulate our hypothesis in "Overview" and describe the registration algorithms employed and validation methods in the remainder of "Methods" section. Next, we present the results generated in "Results and Discussions" and concluding remarks and future directions in "Conclusion".

\section{MATERIALS AND METHODS}

\section{Materials}

As the dual-isotope studies that we are proposing require an additional injection of bone radiotracer, meaning an additional dose to the patient, the imaging study will only be conducted when the feasibility of our approach has been fully proven and that the medical situation of the patient will justify this additional injection. As a result, availability of, and access to, such studies are limited. Nevertheless, we have succeeded in collecting three patient studies. These include two chest studies and one abdominal dual-isotope studies. All 
(a)

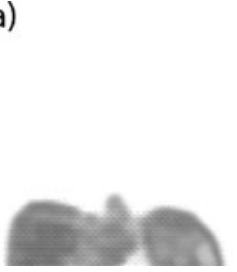

(b)

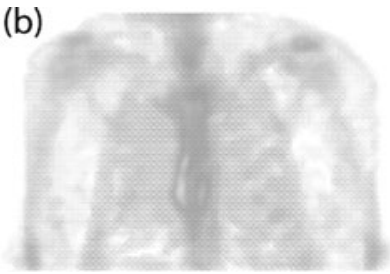

(c)

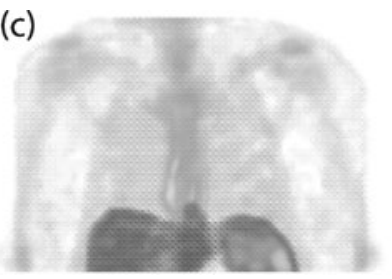

(d)

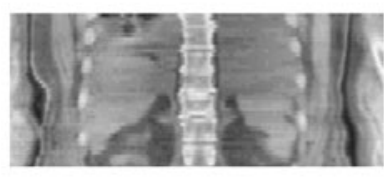

Fig 1. Volume renderings of (a) In-111 SPECT, (b) Tc-99 SPECT, and (c) fused SPECT in a chest study. (d) A coronal slice of the corresponding CT image in the same study.

studies were performed at St. Paul's Hospital (Vancouver, Canada) with a hybrid CT/SPECT system.

All patients were subject to an In-111-labeled white blood cell study for the evaluation of known or suspected infectious processes. Each patient was injected with In-111 a day before imaging and with Tc-99m labeled methylene diphosphonate (MDP) 3-4 h prior to the scans. SPECT/CT studies of both Tc-99m and In-111 were performed after planar imaging using a dual-head camera equipped with a low-power X-ray transmission system (Symbia T6, Siemens). A low-dose CT transmission scan was acquired for $13-16 \mathrm{~s}$ per view with a full FOV consisting of 42-128 slices. After the CT scan, SPECT of a similar FOV was acquired using a $128 \times 128$ matrix $\left(360^{\circ}\right.$ rotation, $15 \mathrm{~s} /$ view $)$ with two $20 \%$ windows centered at $245-\mathrm{keV}$ and $173-$ $\mathrm{keV}$ photopeaks for In-111 and a 10\% window centered at $140-\mathrm{keV}$ photopeak for Tc-99m. More details on the datasets are provided in Table 1.

\section{Methods}

\section{Overview}

Previously proposed image similarity metrics for multimodality registration include statistical correlation and information theory-based measures ${ }^{16,17}$. These have been mostly used for MRI/CT and
PET/CT registrations. In the last decade, mutual information (MI) and normalized mutual information (NMI) have been widely used ${ }^{13,18,19}$ and successfully applied to the registration of brain, thoracic, pelvic, and abdominal images involving different modalities $^{17,20,21}$. The success of MI-based methods is often attributed to its ability to measure the statistical dependence between two images and thus requires no assumption about the explicit relationship between the image intensity values in both modalities. However, when few corresponding image features exist in the images to be registered, optimization of any similarity metrics will fail.

Accordingly, we propose to register CT with Tc-99m SPECT. This is because the MI measured between them will most likely be higher than the MI measured between CT and In-111 SPECT images. This inference can be drawn when we examine the joint histograms created between CT and the individual SPECT images. As shown in Figure $2 \mathrm{a}-\mathrm{c}$, the joint histogram created from CT and In-111 SPECT has the minimal amount of dispersion when misalignment is introduced. Table 2 also provides a comparison of three similarity measures computed between $\mathrm{CT}$ and the individual SPECT images when either no or some misalignment was introduced in the abdominal study. From the table, we see that when the images are in alignment, In-111 SPECT has the lowest values in all measures, meaning that its dissimilarity with $\mathrm{CT}$ is highest.

Table 1. Comparison of Different Similarity Metrics between the Images Before and After Registration

\begin{tabular}{|c|c|c|c|c|c|c|c|c|c|c|}
\hline & \multirow[b]{2}{*}{ NM entropy } & \multicolumn{3}{|c|}{ Joint entropy } & \multicolumn{3}{|c|}{ Mutual information } & \multicolumn{3}{|c|}{ Normalized mutual information } \\
\hline & & Aligned & Misaligned & Difference & Aligned & Misaligned & Difference & Aligned & Misaligned & Difference \\
\hline Tc-99m & 1.557 & 5.83 & 5.0244 & 0.8056 & 0.6477 & 0.0824 & 0.5653 & 1.1111 & 1.0150 & 0.0961 \\
\hline $\ln -111$ & 1.351 & 5.33 & 5.2943 & 0.0357 & 0.4373 & 0.0841 & 0.3532 & 1.0820 & 1.0159 & 0.0661 \\
\hline Fused & 1.914 & 6.00 & 5.8970 & 0.1030 & 0.6610 & 0.0447 & 0.6163 & 1.1105 & 1.0076 & 0.1029 \\
\hline
\end{tabular}

All measures, except NMI, are expressed in bits. The entropy of CT is 4.028 bits 


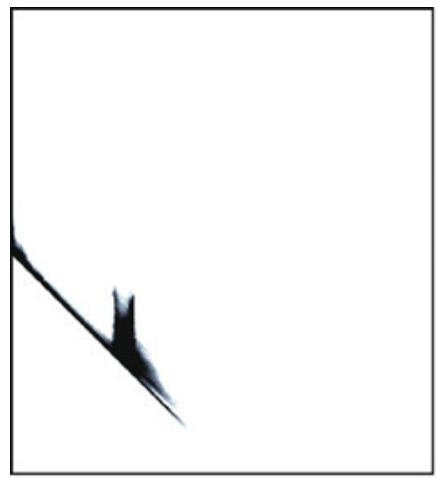

(a)

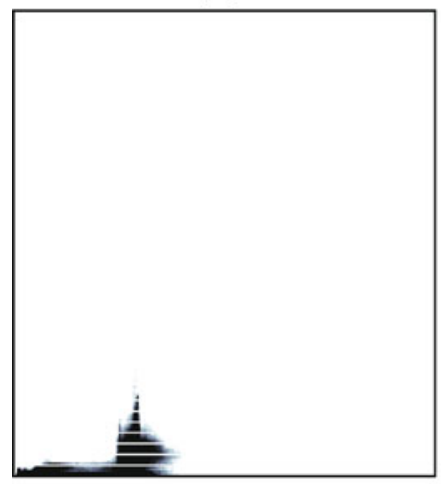

(d)

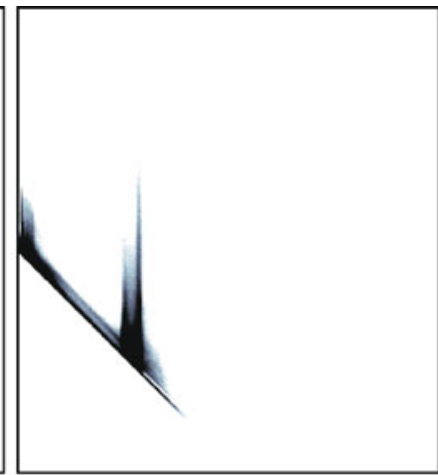

(b)

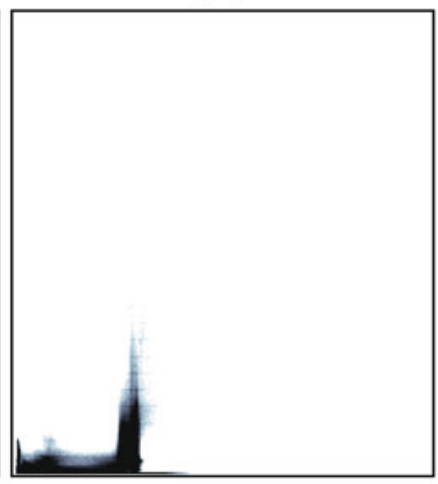

(e)

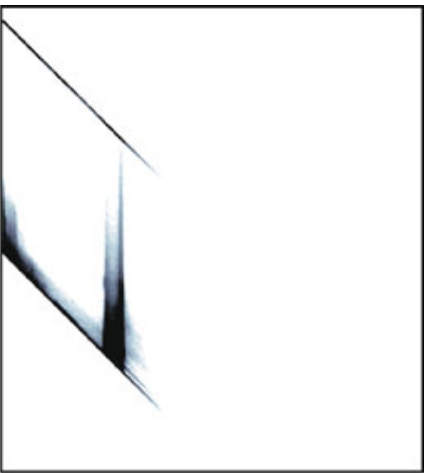

(c)

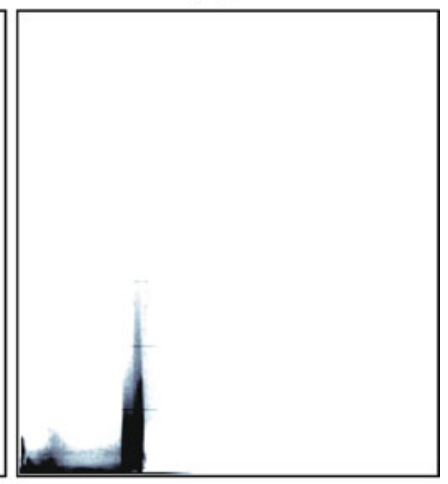

(f)

Fig 2. Joint histograms of CT and a In-111, b Tc-99, and c fused SPECT when misalignment is introduced to the original pairs. $d$, e, $f$ The corresponding histograms at ground-truth position.

Even when misalignment was introduced, the corresponding measures also increased minimally. Lastly, when we inspect the volume renderings of the SPECT images (Fig. 3), we would see that In-111 SPECT has few image features that correspond to the skeletal structures captured in CT (these are depicted as surfaces in Fig. 3d). This clearly supports our hypothesis that Tc-99m SPECT correlates better with CT than In-111 SPECT does. Having noted this increase in correlation, however, one would also question whether combining the information in the individual SPECT images can increase this correlation further. Accordingly, we performed rigid and non-rigid registration trials involving the following three scenarios to quantify the effect of image features on registration accuracies:

1. Scenario1: CT with In-111 SPECT

2. Scenario2: CT with Tc-99m SPECT

3. Scenario3: CT with the fusion of Tc-99m and In-111 SPECT. The fusions of the SPECT images were generated by summing the intensity-normalized versions of Tc-99m SPECT and In-111 SPECT images. An example is shown in Figure 3c.

Note that intensity-normalization was done on the SPECT images because the dynamic ranges of the individual SPECTs are very different. Also, since the implementation of the similarity metrics that we used internally normalize the ranges of the

Table 2. Data Specifications

\begin{tabular}{lll}
\hline & \multicolumn{1}{c}{ Voxel's size $(\mathrm{mm})$} & Image dimensions $(\mathrm{mm})$ \\
\hline Abdomen & & \\
CT & $0.986 \times 0.986 \times 5.0$ & $512 \times 512 \times 128$ \\
SPECT & $4.79 \times 4.79 \times 4.79$ & $128 \times 128 \times 60$ \\
Chest & & \\
CT & $0.699 \times 0.699 \times 5.0$ & $512 \times 512 \times 42$ \\
SPECT & $4.79 \times 4.79 \times 4.79$ & $128 \times 128 \times 70$ \\
\hline
\end{tabular}



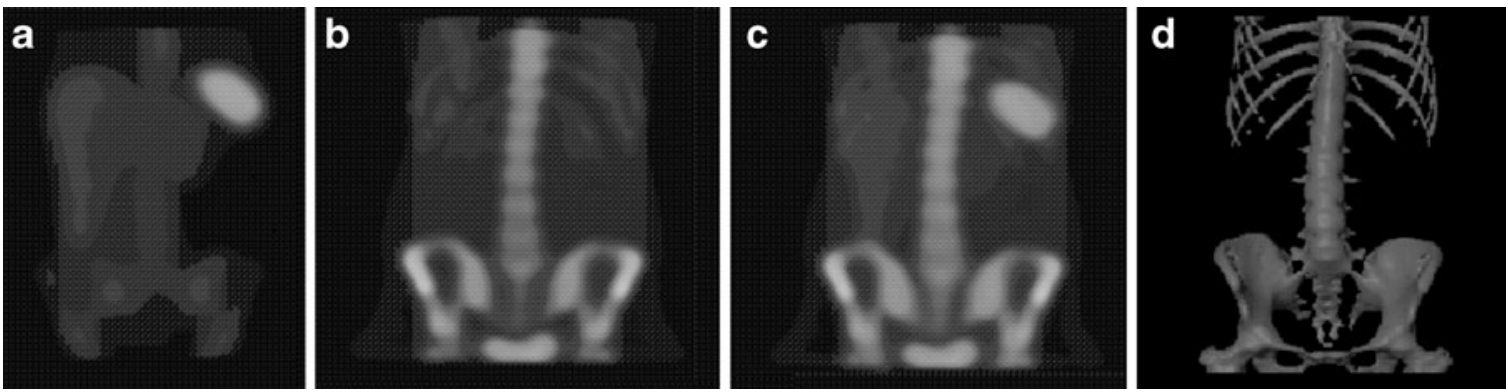

Fig 3. Volume renderings of a In-111, b Tc-99, and c fused SPECT in the abdominal study. d Surface extraction representing the skeletal structures captured in the corresponding CT.

input images, the behaviour of the metric will not be affected by the normalization done on the SPECT images. The latter preprocessing was done so as to equalize their influences on the metric computation. As we intend, the usefulness of this fusion was then later evaluated in our validation tests.

\section{Registration Algorithm}

In all three scenarios, we applied a gradientdescent based optimization algorithm implemented with Insight ToolKit (ITK) ${ }^{16}$ to solve for the misalignment introduced to the data pairs. The registration algorithm (Fig. 4) proceeds in two stages. In the first stage, rigid registration is performed to solve for the six parameters of a rigid-body transformation that would bring the input images into global alignment by minimizing the negative value of $\mathrm{MI}^{22}$ using the versorgradient-descent optimizer ${ }^{16}$. To increase robustness of the algorithm, the transformation is initialized by aligning the principal axes and masses of the input images. A multi-resolution framework was used wherein registration is performed in coarse-to-fine resolutions, taking more samples in a higher resolution image domain and optimizing with smaller step length as we proceed

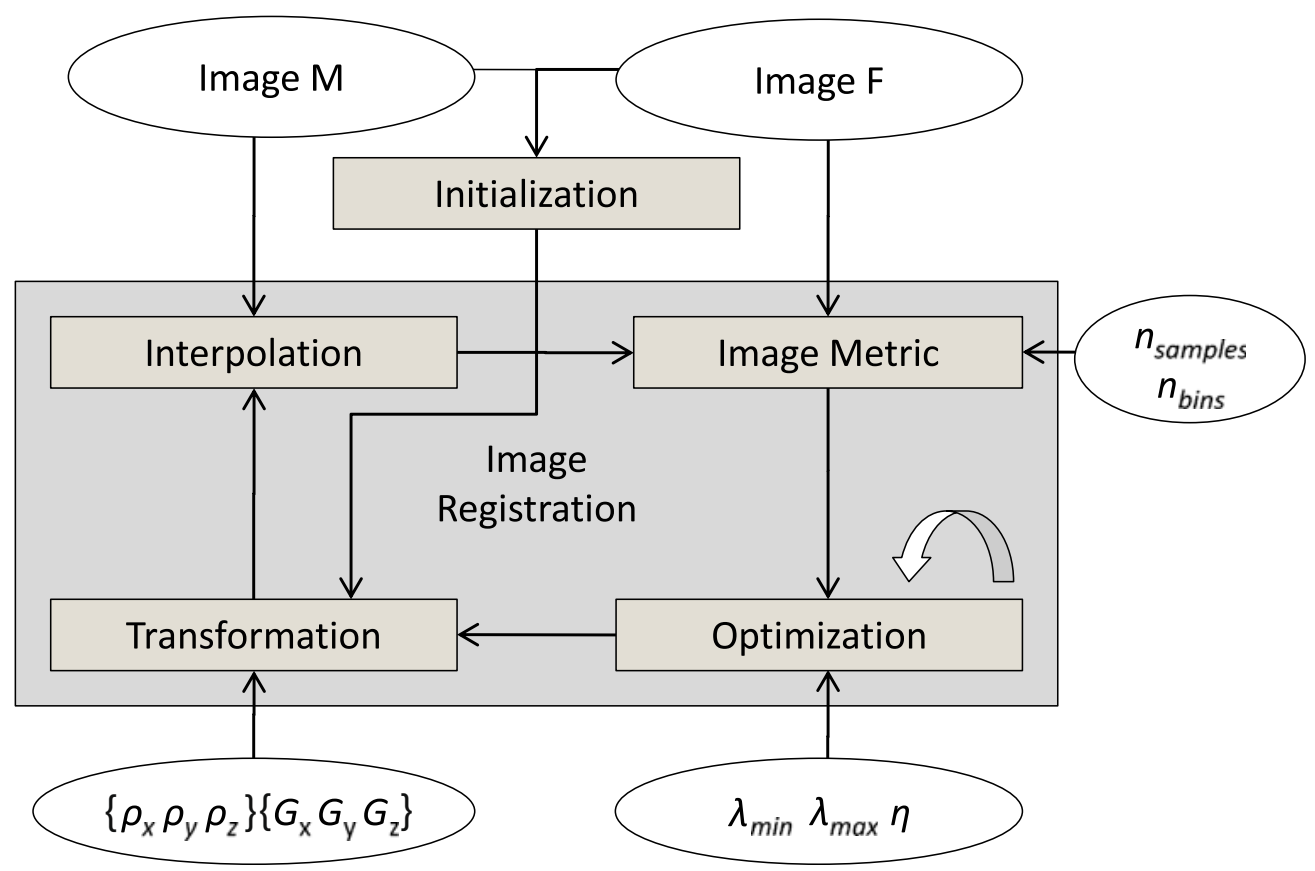

Fig 4. Schematic view of the registration pipeline. Note that when proceeding from rigid to non-rigid registration, the B-spline transformation replaces the rigid-body transformation. 
from one level to the next. According to ${ }^{16,22}$, this effectively decreases the chance of entrapment in local minima and increases the capture range of the algorithm. Finally, to compare against MI, a version of the $\mathrm{NMI}^{16}$ was also tested.

In the second stage, free-form B-spline-based registration was performed in which the linearly registered images are incrementally deformed by manipulating a regular lattice of control points using a third-order B-spline function ${ }^{16}$ by which smoothness constraints are enforced. There exist many regularization techniques in the literature, but we chose B-spline transformation because it has good local shape control capability and high degree of flexibility to deform an image, and thus can better describe local motions that often occur in the anatomical regions involved in this study ${ }^{16}$. In solving for the parameters of each control point, we optimized the MI metric using a variant of the LBGS optimization algorithm ${ }^{16}$. To avoid entrapment in local minima and allow for stable deformations, we also employ a multi-resolution scheme such that at each level of deformable registration, more control points are added by increasing the resolution of the control point grid $\left(G_{x}, G_{y}, G_{z}\right.$ in $x, y, z$ dimensions) and increasing convergence factor $\eta$.

We note here that there are several critical parameters that can impact the success of registration. These include the number of bins $n_{\text {bins }}$ and the number of samples $n_{\text {samples }}$ that are used to compute the MI metric; the scaling of transformation parameters $\rho_{x}, \rho_{y}, \rho_{z}$, which affects the optimization stability ${ }^{15}$; the number of multiresolution levels $n_{\text {levels, }}$, down-sampling factors for SPECT $\left(\alpha_{x}, \alpha_{y}, \alpha_{z}\right)$ and CT $\left(\beta_{x}, \beta_{y}, \beta_{z}\right)$, the optimizer's step length $\left(\lambda_{\min }, \lambda_{\max }\right)$ and the resolution of the control point grid $\left(G_{x}, G_{y}, G_{z}\right)$ all of which determine the fineness of registration at each level. To ensure that a fair comparison of the three scenarios can be done, we thus performed extensive initial experiments to determine the optimal set of registration parameters. These are reported in Tables 3 and 4.

\section{Validation}

As all three datasets were acquired on a hybrid system and that no signs of mis-registration could be observed during careful evaluations of the fused images by two inspectors, the simultaneously acquired Tc-99m and In-111 SPECT images were treated as ground-truth images (i.e. registered to the corresponding CT). Therefore, by applying known spatial transformations (rigid and non-rigid) to the input images and perform registrations on them, we could then compare the accuracies of registration in the three scenarios outlined previously. Specifically, to quantify registration accuracy, a set of landmarks was selected from the CT image in each study for the calculation of the root mean square $(\mathrm{RMS})$ error: $\mathrm{RMS}=\sqrt{\frac{1}{\mathrm{~N}}\left(\mathrm{p}-\mathrm{q}^{2}\right)}$ where $p$ denotes the physical locations of the set of landmarks selected and $q$ denotes the corresponding locations as determined by the registration algorithm.

In this work, we performed two sets of trials; trials where only rigid transformations were introduced to the input images and trials where both global misalignment and non-rigid deformation were introduced. In the first situation, we repeated 80 registrations, each with an initial misalignment consisting of a random translation within a maximum range of $[-50,50] \mathrm{mm}$ in $x$, $y, z$ dimensions and a simultaneous random rotation within a maximum range of $[-15$, 15] degrees along the $x, y, z$ axes. We repeated the same set of trials for each scenario and for each of the two similarity metrics. As we will see in the next section, results from these trials immediately showed us the superiority of MI. While the accuracies of MI and NMI are comparable, MI has a significantly faster computation time. Consequently, in dealing with the more computationally demanding non-rigid registration, we have chosen MI as the similarity metric to be optimized. Similarly, in the second situation where we introduced both rigid transformations and non-rigid deformations, the random rigid body was computed as described before while the B-spline transformation was computed from a set of randomly determined B-spline coefficients. Forty such trials were completed.

\section{RESULTS AND DISCUSSIONS}

All registration trials were performed on a $3.0 \mathrm{GHz}$ PC. On average, rigid and non-rigid registrations took 5 and $12 \mathrm{~min}$, respectively, to 
Table 3. Parameters of Rigid Registration

\begin{tabular}{|c|c|c|c|c|c|c|c|}
\hline \multirow[b]{2}{*}{ Study type } & \multicolumn{3}{|c|}{ Multi-resolution scheme } & \multicolumn{2}{|c|}{ Metric's parameters } & \multicolumn{2}{|c|}{ Optimization } \\
\hline & $\alpha_{x}, \alpha_{y}, \alpha_{z}$ & $\beta_{x}, \beta_{y}, \beta_{z}$ & $n_{\text {levels }}$ & $n_{\text {samples }}(\%)$ & $n_{\text {bins }}$ & $\rho$ & {$\left[\lambda_{\min }, \lambda_{\max }\right]$} \\
\hline Abdomen & $8,8,4$ & $4,4,4$ & 3 & $0.005-0.04$ & $20-64$ & $1.65 e-4-3.65 e-4$ & $0.005-3.0$ \\
\hline Chest & $4,4,4$ & $2,2,2$ & 2 & $0.01-0.03$ & $40-80$ & $1.55 e-3-2.50 e-3$ & $0.001-1.0$ \\
\hline
\end{tabular}

$n_{\text {samples }}$ is expressed as the percentage of total voxels in the volume. $\left\{\alpha_{x}, \alpha_{y}, \alpha_{z}\right\}$ and $\left\{\beta_{x}, \beta_{y}, \beta_{z}\right\}$ are the initial factors by which the input images are down-sampled to.

complete. To quantify the registration errors as a result of the deformations introduced, one of our inspectors also selected points in the CT image as landmarks using a graphical interface that we developed with Fast Light ToolKit. A screenshot of the interface is shown in Figure 5. Registration accuracy is computed as the RMS error between the positions of the landmarks before registration and their positions after registration. To quantify robustness, we also calculated the success rate of different registration trials as the percentage of trials that have RMS errors lower than the SPECT's pixel size of $4.79 \mathrm{~mm}$.

We now discuss the results of the two sets of trials. Registration accuracies of the rigid registrations between the three scenarios are shown in Table 5. From the table, we see that the uses of MI and NMI yielded almost equivalent performances. The registration accuracies in Scenario2 (Tc-99m with CT) are comparable to those in Scenario3 (fused SPECT with CT) while those in Scenariol (In-111 with CT) have the worst performances. Based on analysis of variance (ANOVA), there is significant difference in the registration errors between the three scenarios $(p=2.17 \mathrm{e}-6$, with a $95 \%$ confidence interval), but no significant difference was found between the last two scenarios $(p=0.446,95 \%)$. Furthermore, the number of iterations taken itns in Scenariol is also relatively smaller. This may be explained by examining the MI metric values as shown in Figure 6. Observing closely, we see that the estimated objective function landscape describing the CT and In-111 pair is rougher than the one from CT and Tc-99m (or CT and fused SPECT). The convergence of the optimization in Scenariol may have been due to entrapment in local minima, explaining why itns on average was lower when In-111 SPECT was used.

Registration accuracies are also similar in the cases where rigid and non-rigid misalignment were simultaneously introduced. As shown in the second part of Table 5, the registration errors in Scenario2 are also comparable to those in Scenario3. The RMS errors are highest in Scenario2. With ANOVA, we found significant difference in the errors between the three $(p=0.0094$, with a 95\% confidence interval), and again, no significant difference between the last two scenarios $(p=0.0132,95 \%)$.

Note that, in both sets of trials, the errors are higher in the chest studies than those in the abdominal studies. This may be attributed to the difference between the sizes of the input volumes. From Table 1 and Figure 1c, we can see that CT has a much smaller volume than SPECT does. This difference in sizes and the relatively smaller amount of image features available in the SPECT images have thus created many local minima in the metric space.

Table 4. Parameters of the Non-rigid Registration

\begin{tabular}{|c|c|c|c|c|c|c|c|}
\hline & \multicolumn{5}{|c|}{ Multi-resolution scheme } & \multicolumn{2}{|c|}{ Metric's parameters } \\
\hline & $n_{\text {levels }}$ & $\alpha_{x}, \alpha_{y}, \alpha_{z}$ & $\beta_{x}, \beta_{y}, \beta_{z}$ & $G_{x}, G_{y}, G_{z}$ & & $n_{\text {samples }}(\%)$ & $n_{\text {bins }}$ \\
\hline & & & & $6 \times 6 \times 4$ & $1 e+7$ & $0.01-0.04$ & $20-64$ \\
\hline \multirow[t]{2}{*}{ Abdomen } & 2 & $8,8,4$ & $4,4,4$ & $12 \times 12 \times 8$ & $1 e+1$ & & \\
\hline & & & & $5 \times 5 \times 4$ & $1 e+7$ & $0.02-0.06$ & $40-80$ \\
\hline Chest & 2 & $4,4,4$ & $2,2,2$ & $10 \times 10 \times 8$ & $1 e+1$ & & \\
\hline
\end{tabular}




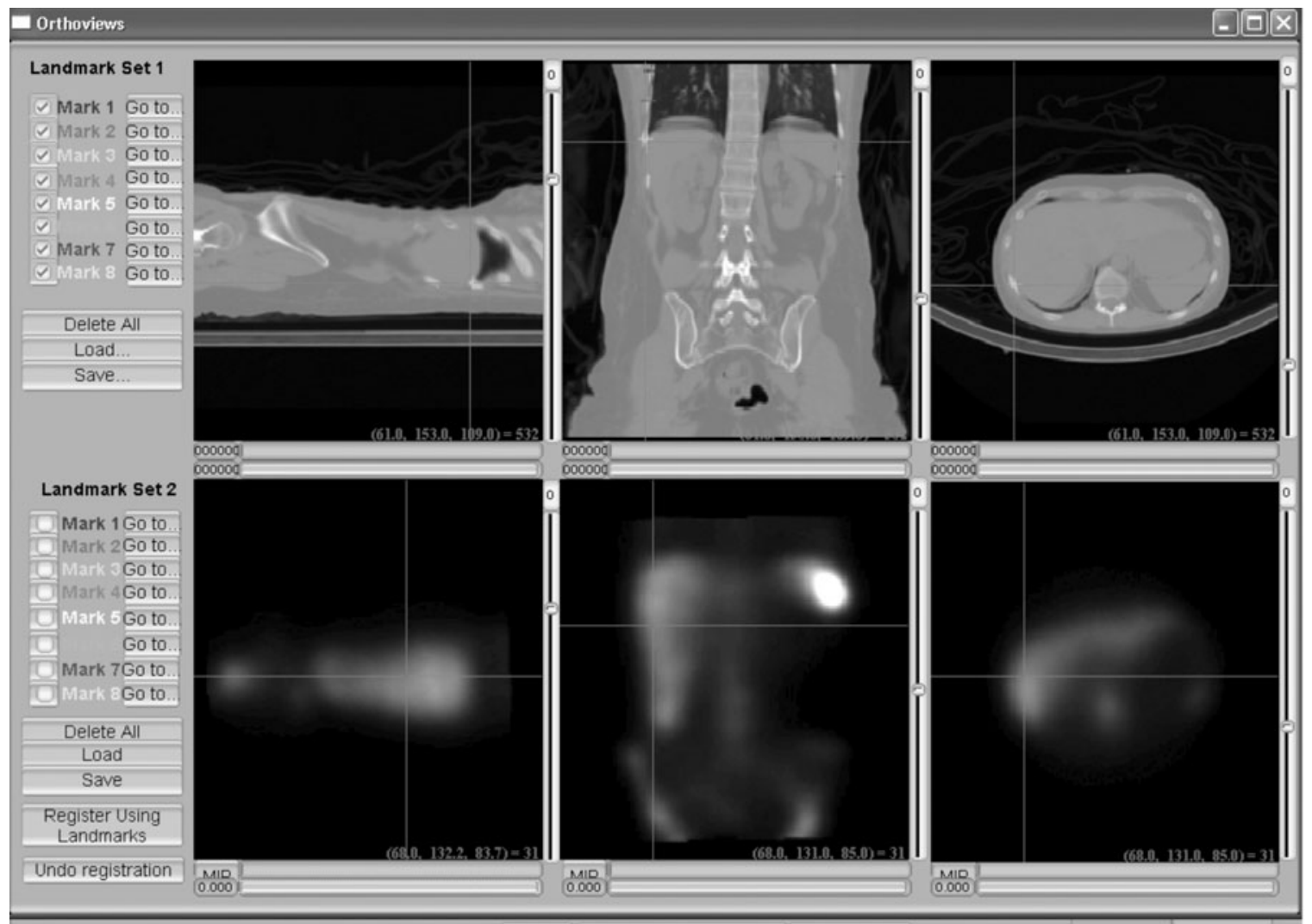

Fig 5. A screenshot of the graphical user interface we developed for the landmarking task preformed for validation of the method.

This further illustrates the importance of having consistent image features in both input images to ensure that the registration algorithm can perform robustly.
In summary, rigid registration of Tc-99m SPECT with CT achieved a success rate of $100 \%$ (success criterion $=\mathrm{RMS}<4.79 \mathrm{~mm}$ ) in the abdominal study and $96 \%$ in the chest studies,

Table 5. Registration Results in Different Scenarios

\begin{tabular}{|c|c|c|c|c|c|c|c|c|c|c|c|c|}
\hline \multirow[b]{3}{*}{ Study } & \multirow[b]{3}{*}{ Scenario } & \multicolumn{8}{|c|}{ Rigid registration } & \multicolumn{3}{|c|}{ Rigid + non-rigid registration } \\
\hline & & \multicolumn{4}{|c|}{ NMI metric } & \multicolumn{4}{|c|}{ MI metric } & \multicolumn{3}{|c|}{ MI metric } \\
\hline & & $\%$ & RMS & itns & Time & $\%$ & RMS & itns & Time & $\%$ & RMS & Time \\
\hline & 1 & 14 & $4.12(0.62)$ & 190 & 6.19 & 15 & $2.99(0.21)$ & 134 & 3.15 & 38 & $4.09(0.28)$ & $3.12+9.03$ \\
\hline & 2 & 90 & $3.87(0.56)$ & 261 & 7.12 & 100 & $3.45(0.63)$ & 221 & 3.59 & 93 & $3.02(0.85)$ & $4.11+12.15$ \\
\hline \multirow[t]{3}{*}{ Abdomen } & 3 & 35 & $3.74(0.24)$ & 279 & 8.56 & 96 & $3.63(0.66)$ & 228 & 4.11 & 87 & $3.16(0.48)$ & $4.51+13.58$ \\
\hline & 1 & 34 & $2.11(0.12)$ & 114 & 6.53 & 8 & $2.91(0.01)$ & 134 & 6.53 & 7 & $2.03(0.36)$ & $5.28+11.33$ \\
\hline & 2 & 82 & $4.33(0.35)$ & 169 & 5.18 & 94 & $3.82(0.29)$ & 175 & 5.18 & 30 & $4.18(0.23)$ & $4.11+15.45$ \\
\hline \multirow[t]{3}{*}{ Chest } & 3 & 80 & $4.12(0.15)$ & 201 & 5.28 & 96 & $3.45(0.78)$ & 218 & 5.28 & 84 & $4.23(0.39)$ & $4.51+14.23$ \\
\hline & 1 & 24 & $3.51(0.14)$ & 190 & 5.18 & 14 & $4.32(0.29)$ & 168 & 5.18 & 21 & $3.29(0.10)$ & $4.42+12.03$ \\
\hline & 2 & 87 & $4.17(0.58)$ & 251 & 5.49 & 94 & $4.06(0.51)$ & 207 & 5.49 & 64 & $3.62(0.91)$ & $3.51+13.15$ \\
\hline Chest & 3 & 87 & $4.12(0.48)$ & 279 & 5.27 & 78 & $3.02(0.21)$ & 191 & 5.27 & 82 & $3.79(0.62)$ & $3.48+14.21$ \\
\hline
\end{tabular}

Success rate $(\%)$ is reported as the percentage of trials with errors lower than the SPECT pixel size of $4.79 \mathrm{~mm}$. The highest success rates in each group are indicated in bold. Standard deviations are shown in parentheses. itns denotes the number of iterations taken before convergence is reached. Time is the average execution time expressed in minutes (including the construction of multi-resolution pyramids). 

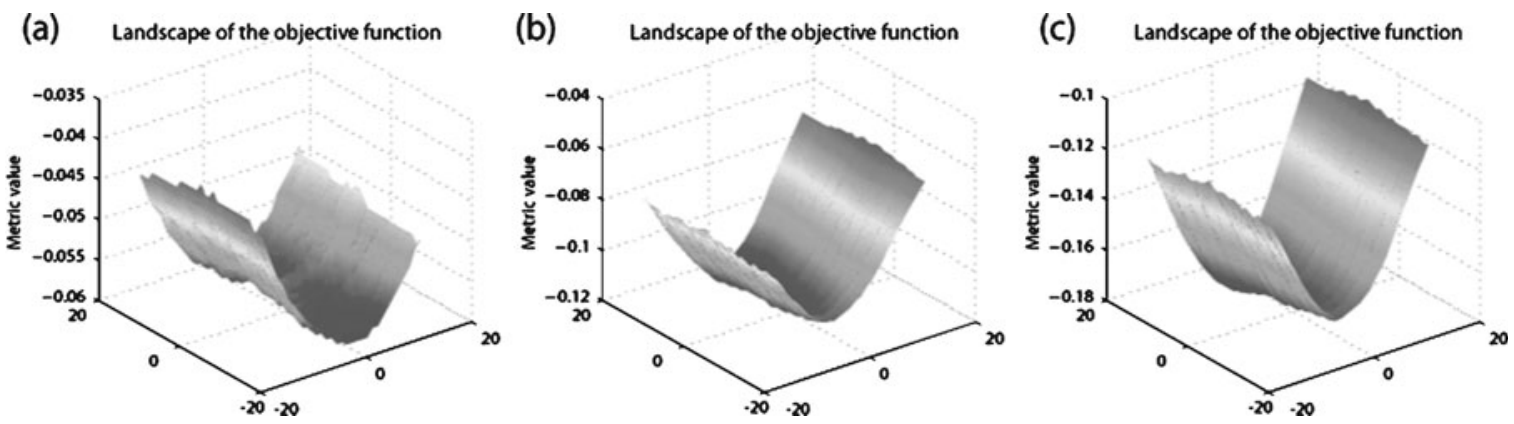

Fig 6. The MI metric plotted against translations in $x$ and $y$ dimensions between (a) CT and In-111, (b) CT and Tc-99m, and (c) CT and fused SPECT.

with average RMS error between 3.45 and $4.45 \mathrm{~mm}$. However, we did not observe higher success rates or accuracy when the fused SPECT was used for registration. Nevertheless, when both global misalignment and local deformations were introduced, success rates were $93 \%$ and $84 \%$ for the abdominal and chest studies, with errors of 3.02 and $4.23 \mathrm{~mm}$, respectively. Conversely, the highest success rate for registration trials of CT and In-111 SPECT images was only $38 \%$. Clearly, the use of Tc-99m SPECT in registration had been effective.

Before we conclude, we note that we did not address the problem of spill-down contamination of the Tc-99m SPECT by the In-111 SPECT. As the focus of the present work is on the accuracy of SPECT-CT registration, we did not account for the quantitative accuracy of the SPECT images. However, for further quantitative analysis of the registered images, scatter reduction should also be applied. In this case, methods as proposed in ${ }^{23,24,27}$ can be used.

\section{CONCLUSION}

In this study, we have performed rigid and nonrigid registrations on $\mathrm{CT}$ and SPECT images that were acquired from dual-isotope acquisitions involving chest and abdominal regions. We have reported the accuracies in the registration of three scenarios, each of which made use of either In-111 SPECT, Tc-99m SPECT, or the fusion of these two images. On a high level, the registration accuracies of fused/Tc- 99m SPECT and CT images are significantly higher than the registration of In-111 SPECT and CT. As In-111 and Tc-99m SPECT are inherently registered, applying the same transformation resolved in the registration of Tc-99m SPECT and CT will immediately give rise to a more accurate alignment of the infection SPECT with CT. Therefore, the use of dualisotope acquisitions has shown to be facilitative and helpful in CT-SPECT registration of infection studies.

Due to the objective of this work, we did not account for errors introduced from data interpolation that might have decreased registration accuracy. In future work, we shall investigate the existence of biased global maximum and local minima due to interpolation ${ }^{24}$. Furthermore, in a much larger patient population, articulated deformations generally exist. Thus, we shall investigate more advanced techniques to deal with cases involving locally rigid $\operatorname{articulations}^{25,26}$ once such medical data is available.

\section{ACKNOWLEDGEMENT}

We would like to thank Dr. Philip Cohen of Pacific Nuclear Medicine Inc., Mathematics of Information Technology and Complex Systems Industrial Postgraduate (MITACS), and Natural Sciences and Engineering Research Council of Canada (NSERC) for providing partial funding of this work and Nazma Tarmohamed, RTNM of St. Paul's Hospital for assisting us in acquiring the image data examined in this paper. 


\section{REFERENCES}

1. Webb A: Introduction to BioMed Imaging. IEEE Press, 2003

2. Heron DE, Smith R, Andrade R: Advances in imageguided radiation therapy - the role of PET-CT. Med Dosimetry $31: 3-11,2006$

3. Roeskea J, Lujana A, Rebab R, Penney B, Yamadac S, Mundta A: Incorporation of SPECT bone marrow imaging into intensity modulated whole-pelvic radiation therapy treatment planning for gynecologic malignancies. Radiother Oncol 77:1117,2005

4. Safaraz M, Wu X, Lodge M, Yu C: Automatic CTSPECT registration of livers treated with radioactive microspheres. Physic Med Biol 49:131-137, 2004

5. Keidar Z, Israel O, Krausz Y: SPECT/CT in tumor imaging. Sem Nucl Med 33(3):205-218, 2003

6. Utsunomiya D, Tomiguchi S, Shiraishi S, Yamada K, Honda T, Kawanaka K, Kojima A, Awai K, Yamashita Y: Initial experience with X-ray CT based attenuation correction in myocardial perfusion SPECT imaging using a combined SPECT/CT system. Ann Nucl Med 19(6):485-489, 2005

7. Hutton B, Braun M: Software for image registration: algorithms, accuracy. Semin Nucl Med 33(3):180-192, 2003

8. Comtat C, Kinahan P, Fessler J: Clinically feasible reconstruction of 3D whole-body $\mathrm{PET} / \mathrm{CT}$ data using blurred anatomical labels. Phys Med Biol 47:1-20, 2002

9. Baete K, Nuyts J, Paesschen W, Suetens P, Dupont P: Anatomical based FDG-PET reconstruction for the detection of hypometabolic regions in epilepsy. IEEE Trans Med Imag 23:510-519, 2004

10. Maintz J, Viergever M: A survey of med image registration. Med Image Anal 2(1):1-36, 1998

11. Zitova B, Flusser J: Image registration methods: a survey. Image Vis Comput 21(11):977-1000, 2003

12. Wells W, Viola P, Atsumi H, Nakajima S, Kikinis R: Multimodal volume registration by maximization of mutual information. Med Image Anal 1:51-53, 1996

13. Maes F, Vandermeulen D, Suetens P: Medical image registration using mutual information. Proc IEEE 91(10):16991722, 2003

14. Tang L, Hamarneh G, Celler A: Coregistration of CTSPECT images for tumor localization and dosimetry. European Association of Nuclear Medicine Annual Congress (EANM), 2006
15. Tang L, Hamarneh G, Celler A: Coregistration of bone $\mathrm{CT}$ and SPECT images using mutual information. In: IEEE International Symposium on Signal Processing and Information Technology (ISSPIT), 2006, pp 116-121

16. Ibanez L, Schroeder W, Ng L, Cates J: The Insight Software Consortium, The ITK software guide, 2006

17. Slomka PJ, Dey D, Przetak C, Aladl UE, Baum RP: Automated 3-dimensional registration of standalone $18 \mathrm{ffdg}$ wholebody PET with CT. J Nucl Med 44(7):1156-1167, 2003

18. West $\mathrm{J}$ : Comparison and evaluation of retrospective intermodality brain image registration techniques. J Comput Assist Tomogr 21:554-566, 1997

19. Slomka P: Software approach to merging molecular with anatomic information. J Nucl Med 45(1):36-45, 2004

20. Shekhar R, Walimbe V, Raja S, Zagrodsky V, Kanvinde M, Wu G, Bybel B: Automated 3-dimensional elastic registration of whole-body PET and CT from separate or combined scanners. J Nucl Med 46:1488-1496, 2005

21. Rizzo G, Arienti R, Castiglioni I, Cattaneo M, Castellone P, Landoni C, Ceresoli G, Messa C, Gilardi MC, Cerutti S, Fazio F: Automatic integration of PET/CT images for clinical use in radiotherapy, in: Engineering in Medicine and Biology Society, 2001. Proceedings of the 23rd Annual International Conference of the IEEE, Vol. 1, 2003, pp 603-606 Vol. 1

22. Mattes D, Haynor D, Vesselle H, Lewellen T: PET-CT image registration in the chest using freeform deformations. IEEE Trans Med Imag 22(1):120-128, 2003

23. Feng J, Penney B: Accurate spill-down and scatter correction for Tc99m/In111 SPECT, Nuclear Science Symposium Conference Record, 2002 IEEE 3 (2002), pp 1562-1566 vol 3

24. Tsao J: Interpolation artifacts in multimodal image registration based on maximization of mutual information. IEEE Trans Med Imag 22(2):854-864, 2003

25. Kovacevic N, Hamarneh G, Henkelman M: Anatomically guided registration of whole body mouse MR images. In: MICCAI, 2003, pp 870-877

26. Huesman RH, Klein GJ, Kimdon JA, Kuo C, Majumdar S: Deformable registration of multimodal data including rigid structures. IEEE Trans Nucl Med 50(3), 2003

27. Shcherbinin S, Celler A, Trummer M, Humphries T: An APD-based iterative reconstruction method for simultaneous technetium-99m/iodine-123 SPECT imaging. IEEE Medical Imaging Conference Record, 2006 